\title{
Analgésicos e antipiréticos isentos de prescrição
}

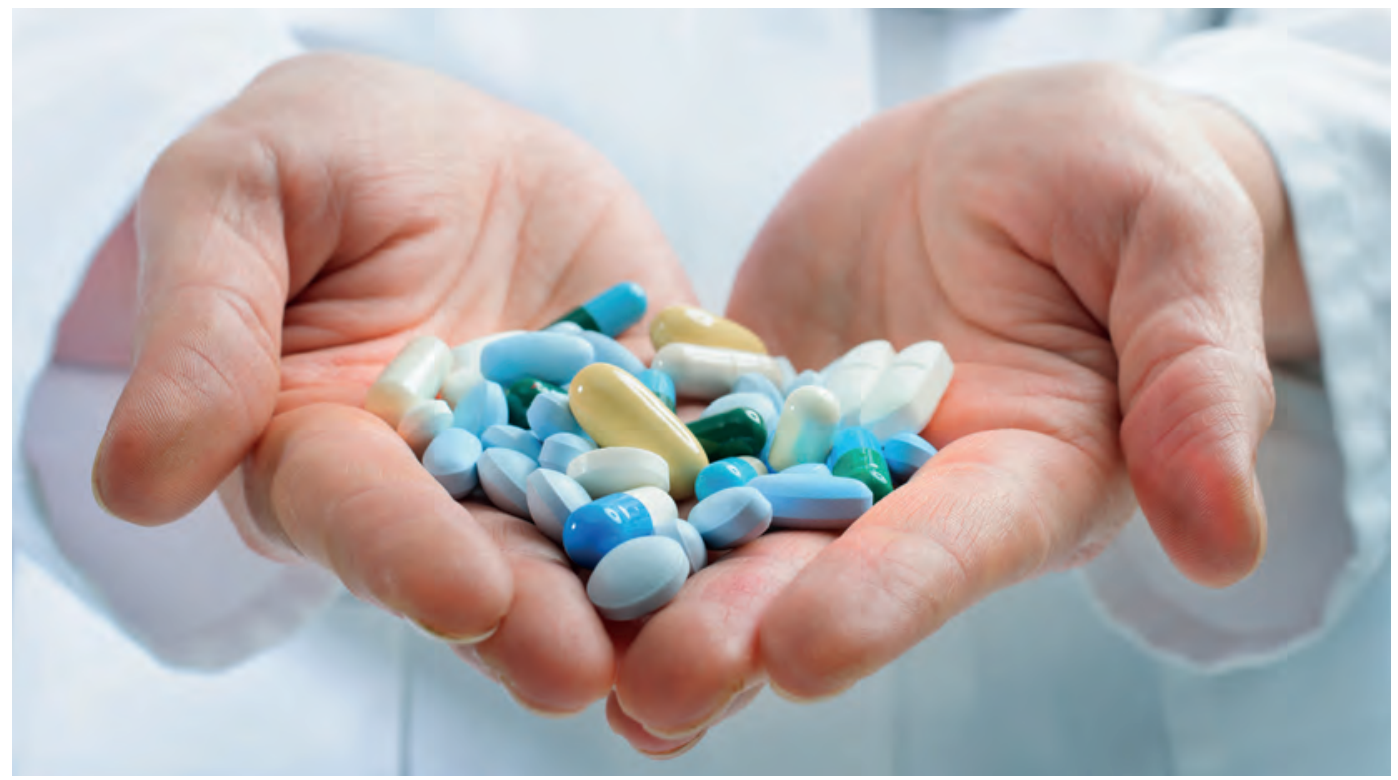

Letícia Nogueira Leite, MSc, PhD Farmacêutica do Centro Brasileiro de Informação sobre Medicamentos/Conselho Federal de Farmácia ORCID: 0000-0002-4490-5165

\section{Introdução}

O uso racional e seguro de medicamentos isentos de prescrição (MIPs) contribui para a prevenção de agravos e para a proteção e promoção da saúde da população. A automedicação, quando bem orientada, pode ser desejável, trazendo beneficio ao usuário de forma ágil e com baixo custo. Entre as principais condições clínicas tratadas por automedicação, citam-se: tosse associada a resfriado, constipação, hiperacidez gástrica, fraqueza generalizada, insônia, infecções de pele, artralgias, queimaduras, dismenorreia, picadas de insetos, cefaleia, dor no corpo e febre?.

De fato, em 2018, um estudo transversal² $(n=270)$ realizado em Ouro Preto/MG, sobre o perfil e fatores associados à automedicação em alunos e servidores do Instituto Federal de Minas Gerais/Ouro Preto, demonstrou que os sinais/sintomas responsáveis pela maior parte dos casos de automedicação foram dor de cabeça/febre (67,9\%), seguidos por aqueles associados a resfriado/gripe (32,6\%). Os MIPs mais utilizados foram os analgésicos/antipiréticos $(65,8 \%)$, seguidos pelos "antigripais" (21,4\%) e os anti-inflamatórios (18,7\%). O motivo mais apontado para a prática da automedicação foi a experiência anterior com o medicamento utilizado (54,5\%).

Segundo a Instrução Normativa (IN) n 86 da Agência Nacional de Vigilância Sanitária (Anvisa), que instituiu a Lista de Medicamentos Isentos de Prescrição (LMIP), os analgésicos e 
antipiréticos são indicados para o tratamento de pessoas com dores leves a moderadas, incluindo as associadas com sinais/sintomas da gripe e resfriado e com cólicas menstruais, enxaqueca, febre, cefaleia, dores musculares e articulares, e dor após traumas 3 .

Como qualquer medicamento, os analgésicos e antipiréticos isentos de prescrição oferecem riscos de danos à saúde quando utilizados de forma inadequada e sem a orientação de profissional habilitado. A automedicação é potencialmente mais segura e efetiva quando acompanhada de orientação adequada ao paciente, uma vez que isso reduz o risco de erros e promove o uso seguro e racional de medicamentos.

Neste artigo, será discutido o uso racional e seguro de analgésicos e antipiréticos isentos de prescrição para alívio dos sinais/sintomas da dor e da febre, considerando também as terapias não farmacológicas.

\section{Dor e Febre}

\section{Dor}

A dor é sintoma comum a muitos quadros clínicos, como manifestação direta de um trauma ou parte da evolução de uma doença. A Associação Internacional para o Estudo da Dor (International Association for the Study of Pain - IASP) define dor como: "Experiência sensorial e emocional desagradável, relacionada com lesão tecidual real ou potencial, ou descrita em termos deste tipo de dano"4.

O impacto da dor é substancial, especialmente quando intensa e persistente, associando-se a alterações cognitivas e emocionais (depressão, ansiedade, temor, diminuição da atividade sexual), alterações físicas (náuseas, perda de apetite, transtornos do sono e dimi- nuição da força e da resistência), redução da qualidade de vida, prejuízo na realização de atividades diárias e laborais, repercussões sociais e econômicas ${ }^{4,5}$.

\section{Classificações dos tipos de dor}

A dor pode ser classificada segundo critérios topográficos (localizada e generalizada; superficial, somática profunda e visceral), fisiopatológicos (orgânica e psicogênica), de intensidade (leve, moderada e intensa) e temporais (aguda e crônica). A seleção do grupo farmacológico de analgésicos está diretamente relacionada à intensidade da dor relatada pelo paciente. A Organização Mundial da Saúde (OMS) sugere escalonamento do tratamento da dor, de acordo com a percepção de sua intensidade pelo paciente: para dor leve, analgésicos não opioides (ex.: dipirona, paracetamol e anti-inflamatórios não esteroides - AINEs); para dor moderada, sugere-se associar opioide fraco (ex: tramadol e codeína) a agente não opioide; e, para dor intensa, usam-se opioides fortes (ex.: morfina e metadona) $)^{4,6}$.

Com o intuito de auxiliar a análise semiológica, foram desenvolvidas diversas metodologias que avaliam a intensidade da dor e ajudam na escolha do medicamento adequado. As escalas verbais consistem em perguntar ao paciente qual é a intensidade da dor que ele está sentindo no momento. As respostas podem ser: sem dor, dor leve, dor moderada, dor intensa ou a pior dor possível. A escala analógica visual consiste numa linha horizontal que tem assinalada numa extremidade a classificação "leve" e, na outra, a classificação “intensa”. O paciente deve indicar em que parte da régua encontra-se sua dor. Mede-se a distância entre o início da linha, que corresponde a zero, e o local assinalado, obtendo-se, assim, uma classificação numérica da dor que poderá auxiliar no manejo?. 


\section{Cefaleias}

As cefaleias, as sindromes dolorosas miofasciais, a fibromialgia, as artropatias, e as doenças osteomusculares relacionadas ao trabatho, são as razões mais comuns da procura por atendimento nos sistemas de saúde relacionado à dor ${ }^{5}$. De fato, as cefaleias são um problema comumente relatado em pesquisas de todo o mundo, causando problemas de saúde e incapacitação. Estudos epidemiológicos têm buscado estimar a sua prevalência em diferentes populações e o seu impacto, tanto na população como nos sistemas de saúde. A prevalência da cefaleia ao longo da vida é elevada (maior que 90\%) e a taxa de prevalência anual é estimada em 46\% na população geral adulta ${ }^{8}$.

A cefaleia pode ser primária ou secundária. As cefaleias primárias são doenças cujo sintoma principal, porém não o único, é a dor de cabeça (ex:: migrânea, cefaleia do tipo tensional e cefaleia em salvas). As cefaleias secundárias são o sintoma de uma doença subjacente, neurológica ou sistêmica (ex.: meningite, dengue, tumor cerebral) 9 .

A cefaleia do tipo tensional (CTT) é um tipo comum de cefaleia primária, com prevalência ao longo da vida entre $30 \%$ e $78 \%$, observada em diferentes estudos. Pode ser classificada em três subtipos, de acordo com a frequência da cefaleia: CTT episódica infrequente (menos de um cefaleia por mês), CTT episódica frequente (uma a 14 cefaleias por mês) e CTT crônica (15 ou mais cefaleias por mês) ${ }^{9}$. A CTT caracteriza-se por crises de cefaleia de intensidade leve a moderada, geralmente descritas como uma pressão ou aperto (não pulsátil), que não pioram com a atividade física rotineira, como caminhar ou subir escadas. A dor se mantém por horas a dias e, na maioria das vezes, é bilateral ${ }^{9}$.

\section{Cefaleia por uso excessivo de medicamentos}

O uso impróprio de medicamentos é a causa de diversos agravos à saúde. Há nove anos, publicamos um artigo neste boletim sobre questões de segurança da automedicação com analgésicos e a cefaleia ocasionada por uso excessivo de medicamentos ${ }^{10}$. Naquela ocasião, já era apontada a importância da educação da população sobre autocuidados para cefaleia e sua relevância para a saúde pública.

A cefaleia por uso excessivo de medicamentos (CEM) caracteriza-se pelo aumento da frequência ou da intensidade da cefaleia, em pacientes que sofrem de alguma cefaleia primária preexistente (especialmente migrânea e/ou CTT). Ocorre em mais de 15 dias ao mês, por um período mínimo de três meses, como consequência de uso excessivo de medicamentos para dor de cabeça aguda ou sintomática. Os medicamentos de uso excessivo podem ser analgésicos comuns (ibuprofeno, paracetamol e ácido acetilsalícilico), analgésicos combinados (contendo cafeína e outras substâncias adicionadas), ergotamínicos, triptanos ou opioides, desde que tomados regularmente (mais de 10 ou 15 dias por mês, dependendo do medicamento $)^{9}$.

Evidências epidemiológicas de muitos paises indicam que mais da metade das pessoas com dor de cabeça, em 15 dias ou mais por mês, têm CEM. Pacientes com outros quadros de dor (dor musculoesquelética crônica e doenças reumáticas) também podem desenvolver CEM devido ao uso diário de analgésicos, especialmente se já tiverem história de cefaleia primária9,11.

A fisiopatologia da CEM ainda não está claramente compreendida, mas sensibilização central, fatores genéticos, alterações endócrinas e mecanismos psicológicos (estratégias de lidar com a dor, aprendizado e fatores 
comportamentais) podem estar envolvidos. O risco de desenvolvimento dessa cefaleia é diferente para as diferentes substâncias e parece ser maior com ergotamínicos, opioides, triptanos e analgésicos combinados, em comparação aos analgésicos simples ${ }^{11}$.

A maioria dos pacientes com esse distúrbio melhora após a interrupção abrupta ou gradual do analgésico em uso excessivo. A prevenção é especialmente importante em pacientes com tendência a cefaleias frequentes s,11. $^{9,1}$

Em vários estudos, a taxa de recorrência após a interrupção do medicamento em abuso foi de até $30 \%$ em um ano. Portanto, após a interrupção do uso do medicamento causador da CEM, os pacientes devem ter um seguimento regular, com acompanhamento multidisciplinar, para evitar a recorrência. O aconselhamento ao paciente sobre as causas e consequências da CEM, pelo profissional da saúde, é parte essencial do manejo e pode ser feito com sucesso na atenção primáriaa ${ }^{9,11}$.

\section{Automedicacação para tratamento da dor}

A automedicação é uma realidade entre as pessoas com dor de cabeça. Em um inquérito realizado por telefone com 274 pessoas que apresentavam dor de cabeça frequente, apenas $1 \%$ utilizava medicamento prescrito por profissional habilitado. A maioria das pessoas utilizava analgésicos isentos de prescrição (56\% usavam paracetamol e 15\% ácido acetilsalicílico) e a eficácia percebida dos analgésicos isentos de prescrição utilizados foi de aproximadamente 7 em uma escala de 0 a $10^{12}$.

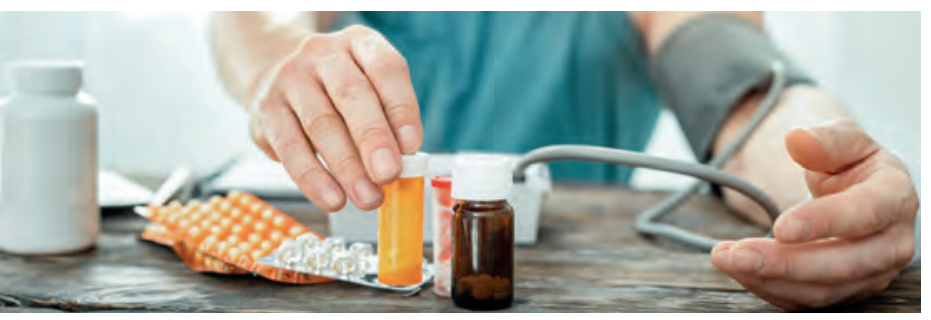

\section{Febre}

A febre perfaz cerca de $30 \%$ das consultas pediátricas e $20 \%$ das queixas em serviços de urgência, na população geral. Não é um sinal isolado e pode incluir vários sinais/sintomas que resultam da resposta de fase aguda, com produção de citocinas inflamatórias, alterações metabólicas e circulatórias. A apresentação típica inclui: astenia, anorexia, sonolência, cefaleias, delírio, mialgias, tremores, artralgias, náuseas, vômitos, convulsões, desidratação, taquicardia, taquipneia e lesões herpéticas ${ }^{13,14}$.

A regulação da temperatura corporal requer um equilíbrio entre a perda e a produção de calor. O hipotálamo, que regula o ponto de configuração no qual a temperatura corporal é mantida, é formado por uma porção anterior da área pré-óptica, constituída por células nervosas sensiveis à temperatura. Duas áreas destacam-se nesta porção: uma anterior, que provoca maior dissipação de calor, e outra posterior, que gera e conserva o calor. As vias eferentes do centro dissipador promovem vasodilatação periférica e aumento da sudorese, enquanto as vias do centro gerador de calor promovem vasoconstrição periférica e aumento da atividade metabólica corporal (via suprarrenal e atividade simpática), além do aumento do tônus muscular (calafrios)15,16.

Na febre, há uma elevação do ponto de ajuste da temperatura corporal, decorrente da ação de prostaglandinas do tipo E no hipotálamo. A sintese aumentada de prostaglandinas do tipo E é estimulada pela formação de citocinas

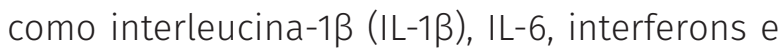
fator de necrose tumoral- $\alpha$, liberados em diversas situações, como, por exemplo, em infecções e lesões teciduais ${ }^{15,17}$.

A temperatura corporal varia conforme o local e o momento da mensuração. Quando a 
mensuração é feita na cavidade oral, a temperatura considerada normal é de $36^{\circ} \mathrm{C}$ a $37,8^{\circ} \mathrm{C}$; para a mensuração no reto, a temperatura é, em média $0,6^{\circ} \mathrm{C}$ maior. A temperatura axilar normal varia entre $36^{\circ} \mathrm{C}$ e $37,4^{\circ} \mathrm{C}$. O estado de febre pode ser definido como temperatura oral acima de $37,2^{\circ} \mathrm{C}$, pela manhã, ou acima de $37,7^{\circ} \mathrm{C}$, à tarde. Existem variações de temperatura corpórea de acordo com o turno, sendo observados niveis mais baixos pela manhã e mais elevados ao final da tarde. Vários processos fisiológicos também podem influenciar a temperatura corpórea, destacando-se o envelhecimento, o ciclo menstrual, a gravidez e a atividade física ${ }^{16,18}$.

As principais afecções clínicas que podem vir acompanhadas de febre são: laringite, faringoamigdalite, otite média, distúrbios metabólicos, desregulação do sistema nervoso central e estados de imunodeficiência, infecções bacterianas, virais ou fúngicas, leishmaniose visceral, malária, infecção pelo vírus da zica, da gripe, da dengue, da chicungunha e da febre amarela. Também é comum a ocorrência de febre pós-vacinal. Além disso, alguns medicamentos podem causar elevação da temperatura corporal por diversas razões, entre as quais, a alteração do mecanismo de termorregulação ${ }^{19}$.

Apesar da ampla variedade do espectro clínico, conhecer o padrão de manifestação de algumas afecções associadas a quadro febril pode ser útil para a avaliação da gravidade e a identificação da necessidade de encaminhamento do paciente a um médico. O conhecimento dos mecanismos envolvidos na ocorrência de febre e as suas consequências biológicas é importante pela sua utilidade na interpretação semiológica e na tomada de decisão clínica ${ }^{19}$. O guia de prática clínica "Sinais e sintomas não específicos: febre"19, publicado em 2018 pelo Conse-
Iho Federal de Farmácia (CFF), fornece as bases para que o farmacêutico selecione a melhor conduta quando apoia o paciente no controle da febre, apresentando informações sobre anamnese farmacêutica e verificação de parâmetros clínicos, situações especiais e precauções, alertas para encaminhamento, elaboração do plano de cuidado (terapia não farmacológica e terapia farmacológica com medicamentos isentos de prescrição) e avaliação dos resultados. O guia pode ser acessado, gratuitamente, em: https:/ / bit.ly/2StwR2w

\section{Prevenção e terapia não farmacológica para dor e febre}

A prevenção da dor baseia-se em evitar, quando possivel, as atividades responsáveis por desencadeá-la. As cefaleias, por exemplo, podem surgir devido a uma má noite de sono ou a gatilhos como chocolate, cafeína, odores fortes, entre outros ${ }^{20}$. As lesões musculares responsáveis por causar dor podem ser evitadas pela realização de alongamento e aquecimento antes da prática de exercícios físicos, por exemplo. Tais lesões também podem ser evitadas por meio de correção postural e aplicação de cargas e movimentos adequados ao estado de condicionamento físico do indivíduo ${ }^{21}$.

O tratamento da dor deve priorizar, quando possivel, a eliminação dos elementos que ativam e sensibilizam as vias nervosas. Porém, como nem sempre isso é possivel, algumas estratégias terapêuticas, como terapias psicológicas, fisioterapia (para dores que envolvam o sistema musculoesquelético, visando reduzir a dor, corrigir postura, melhorar equilíbrio e reforçar musculatura) e acupuntura, podem ser empregadas para minimizar a dor 4,5

Outras medidas podem ser utilizadas para reduzir a dor, como: aplicação de frio em casos 
de dor musculoesquelética, torção ou contusão; aplicação de calor quando ocorrem espasmos musculares; sono regular; evitar bebidas alcoólicas; controle de estresse e lazer. Também são úteis a realização de exercícios físicos ou o repouso, dependendo da etiologia ${ }^{5,22}$. As estratégias terapêuticas devem ser adaptadas para cada caso, de acordo com a evolução relatada pelo paciente ${ }^{11}$.

Para o controle da febre preconiza-se promover alívio ao paciente (especialmente a criança) com medidas físicas de resfriamento (banhos de esponja ou imersão com água morna, e resfriamento do ambiente), facilmente disponiveis e de baixo custo. O objetivo é aumentar a perda de calor e, consequentemente, diminuir a temperatura corporal ${ }^{19,23}$. Todavia, é importante ter em mente que a febre é um processo importante na ativação de mecanismos naturais de defesa do organismo, por isso, não deve ser inibida de forma indiscriminada.

Uma revisão sistemática Cochrane ${ }^{24}$, envolvendo sete ensaios clínicos randomizados e quasi-randomizados (467 participantes), avaliou diferentes estratégias não farmacológicas em crianças com febre de origem infecciosa presumida. Os métodos físicos não diferiram do placebo em eficácia ou segurança. Um pequeno estudo ( $n=30$ ), que comparou métodos fisicos (esponja com água morna) ao grupo placebo não demonstrou diferença na proporção de crianças sem febre, uma hora após o tratamento. Porém, na segunda hora, a febre havia desaparecido em 8 dos 15 participantes no grupo de esponja com água morna versus nenhum dos participantes do grupo placebo (0/15) [Risco Relativo (RR): 17; Intervalo de Confiança de 95\%(IC95\%): 1-270]. O amplo intervalo de confiança reflete o limitado tamanho da amostra no estudo. Em dois ensaios, em que todas as crianças receberam paracetamol, os métodos fisicos resultaram em maior proporção de crianças sem febre em uma hora $[\mathrm{n}=125$; RR:11,8 (IC95\%: 3,4-40,8)]. Um terceiro estudo ( $n=130)$ não encontrou diferenças entre os grupos. Eventos adversos leves (tremores e arrepios) foram mais comuns nos grupos que receberam métodos físicos do que nos que tomaram apenas paracetamol [(RR: 5 (IC95\%: 1,6-16,6)].

Recomenda-se que os métodos físicos sejam realizados em conjunto com o uso de antipiréticos, pois, isoladamente, têm pouca efetividade. Os métodos físicos podem ser úteis enquanto se aguarda o período de latência do antipirético ${ }^{19,23}$.

Outras condutas importantes incluem: utilizar roupas leves, não cobrir demais o paciente, manter o ambiente fresco e ingerir líquidos para evitar desidratação. Friccionar álcool na pele e aplicar bolsas de gelo também têm sido usados para resfriar o corpo durante a febre, mas não são recomendados, uma vez que esses métodos podem causar efeitos adversos graves ${ }^{19,24}$.

\section{Tratamento farmacológico}

Para a definição de conduta apropriada para o controle da dor e da febre, é indispensável que se considere certos critérios na seleção da terapia farmacológica (Quadro 1). A seleção de um medicamento também deve levar em conta as possiveis interações do medicamento selecionado com outros medicamentos em uso pelo paciente, bem como com alimentos, tabaco e álcool ${ }^{19}$.

Os analgésicos não opioides são os medicamentos mais comumente utilizados no tratamento de dores leves a moderadas, agudas e crônicas, e da febre. Entre eles, incluem-se paracetamol, dipirona, ácido acetilsalicílico e 
Quadro 1. Seleção da terapia farmacológica para o alívio da dor ou febre.

1. Eficácia superior demonstrada para desfechos clínicos relevantes, frente a alternativas terapêuticas, farmacológicas ou não;

2. Segurança superior demonstrada frente a alternativas terapêuticas, farmacológicas ou não, mensurada por menores frequência e gravidade de reações adversas e pelo perfil de contraindicações, considerando necessidades e características individuais do paciente;

3. Conveniência de forma farmacêutica e de esquema terapêutico, considerando idade, habilidades e rotina do paciente;

4. Menores frequência e gravidade de potenciais interações indesejáveis com outros medicamentos, alimentos, tabaco e álcool;

5. Acesso mais fácil (baixo custo ou distribuição gratuita pelo sistema público de saúde);

6. Preferência do paciente por um dado medicamento, em função de sucesso terapêutico e efeitos adversos experimentados previamente.

Fonte: adaptado de Fuchs ${ }^{4}$.

demais AINEs. Todos têm propriedades analgésica e antipirética. Paracetamol e dipirona são comumente agrupados entre os AINEs, mas sua fraca atividade anti-inflamatória não tem repercussão clínica 4,19.

Os AINEs produzem efeito anti-inflamatório, antipirético e analgésico por inibição da cicloxigenase (COX) e, assim, impedem a produção de prostaglandinas e tromboxanos que estão diretamente relacionados com os processos de febre, inflamação e dor ${ }^{25}$. Entretanto, é importante considerar o tipo de dor, bem como sua intensidade, na avaliação da eficácia analgésica. São particularmente eficazes quando uma inflamação desencadeia a sensibilização dos receptores de dor a estímulos mecânicos e químicos normalmente indolores ${ }^{15}$. Aproximadamente $60 \%$ dos pacientes respondem ao AINE utilizado. Para os $40 \%$ restantes, recomenda-se sua substituição por representante de diferente classe química, pois o fato de não terem respondido a um AINE não implica ausência de resposta aos demaišs.

Já no controle da febre, os AINEs promovem o retorno da temperatura corporal ao nível normal. Porém, esses fármacos não influenciam a temperatura corporal quando ela está elevada por fatores como exercício ou em resposta à temperatura ambiente ${ }^{15}$.

Os medicamentos para o alívio da febre e da dor, cuja venda é isenta de prescrição, são: ácido acetilsalicílico, ibuprofeno, paracetamol, naproxeno, diclofenaco e dipirona. Importante salientar que algumas apresentações dos fármacos anteriormente citados podem requerer prescrição médica, em razão da dosagem mais elevada (ex: ibuprofeno 600 mg) ou da forma farmacêutica (ex: diclofenaco de uso sistêmico). A seguir, são apresentados os MIPs utilizados para tratar dor e febre, bem como um sumário 
das evidências quanto à segurança e eficácia, situações especiais e contraindicações (Quadro 2).

Ácido acetilsalicílico. O ácido acetilsalicílico é um inibidor potente da síntese de prostaglandinas e da agregação plaquetária. Inativa irreversivelmente a COX, atuando tanto sobre a COX-1 quanto sobre a COX-217,26. É uma alternativa ao paracetamol, com similar eficácia analgésica e antipirética ${ }^{27}$, amplamente empregado ao redor do mundo por seu baixo custo; no entanto, sua menor segurança tem levado à redução do consumo em países desenvolvidos4.

Não é habitualmente indicado para crianças e adolescentes com menos de 16 anos, em razão da associação causal com a síndrome de Reye em crianças com infecções virais (Influen$z a$, herpes ou varicela-zoster) $)^{19,28}$. A sindrome de Reye é uma encefalopatia rapidamente progressiva com disfunção hepática, caracterizada por vômitos e confusão, com rápido decurso para convulsões e coma. O risco de síndrome de Reye parece ser aumentado em crianças e jovens com sinais/sintomas de infecção viral, tratados com ácido acetilsalicílico ${ }^{19}$. Além disso, medicamentos contendo ácido acetilsalicílico merecem atenção especial quando prescritos para pacientes que vivem em áreas endêmicas de dengue, zica e chicungunha ou que tenham outras infecções, pois há a possibilidade de ocorrência de sangramento. Nessas situações, são contraindicados ${ }^{19}$.

Uma revisão sistemática ${ }^{29}$, envolvendo 67 ensaios clínicos randomizados, duplo-cegos e controlados por placebo ( $n=5743$ participantes), avaliou a eficácia e a segurança do ácido acetilsalicílico, em dose única, no alívio da dor pós-operatória de intensidade moderada a grave. As buscas envolveram as bases de dados Medline, The Cochrane Library, Oxford Pain Relief Database e Embase, com artigos publicados até ja- neiro de 2012. Para o desfecho "pelo menos 50\% de alívio da dor em três a seis horas, sem uso de medicação resgate", após tratamento com dose única de ácido acetilsalicílico 600/650 mg, 900/1000 mg e 1200 mg, foram obtidos os seguintes resultados, respectivamente: número necessário para tratar (NNT) de 4,2 (IC95\%: 3,9-4,8), $\mathrm{RR}=2,5$ (IC95\%: 2,2-2,7); NNT=3,8 (IC95\%: 3,0-5,1), $\mathrm{RR}=2,7$ (IC95\%: 2,0-3,7); e NNT=2,7 (IC95\%: 2,0-3,8), $\mathrm{RR}=2,9$ (IC95\%: 2,0-4,2). Dose única de ácido acetilsalicílico $900 \mathrm{mg} / 1000 \mathrm{mg}$ produziu mais sonolência, tontura, náusea e irritação gástrica que placebo, com número necessário para produzir dano (NND)=7,5 (IC95\%: 4,8-17). Doses mais altas do ácido acetilsalicílico demonstraram menor NNT, porém, merece atenção o fato de também estarem associadas a aumento de efeitos adversos, incluindo sonolência e irritação gástrica, quase todos transitórios e de gravidade leve a moderada.

Diclofenaco. Os AINEs tópicos são formulados para aplicação direta no local da dor, evitando a distribuição e seus consequentes efeitos adversos sistêmicos. Este método de aplicação necessariamente limita seu uso a condições dolorosas mais superficiais, como entorses, distensões e dores musculares ou tendinosas. Eles não são apropriados para uso em pele ferida. Uma revisão sistemática Cochrane ${ }^{30}$, envolvendo 61 ensaios clínicos randomizados, duplo-cegos ( $n=8.386$ participantes), avaliou a eficácia e a segurança de diferentes formas farmacêuticas (gel, creme, emplastro e spray) de AINEs aplicados topicamente para tratar adultos com dor musculoesquelética aguda. As buscas envolveram as bases de dados Medline, Cochrane Register of Studies Online e Embase, com artigos publicados até fevereiro de 2015. As condições clínicas tratadas foram entorses, distensões, contusões ou lesões por uso excessivo, como tendinite. 
Diclofenaco, ibuprofeno, cetoprofeno, piroxicam e indometacina foram mais eficazes que o placebo para o desfecho sucesso clínico, definido como pelo menos 50\% de alívio da dor (evidências de qualidade moderada ou alta). Para o diclofenaco tópico (10 estudos analisados; 2050 participantes), o RR foi de 1,6 (IC95\%: 1,5-1,7) e o NNT para alcançar pelo menos $50 \%$ de alívio da dor foi de 3,7 (IC 95\%: 3,2-4,3). Ou seja, a cada quatro participantes que aplicaram diclofenaco tópico, um teve redução de pelo menos $50 \%$ na intensidade da dor.

$\mathrm{Na}$ análise comparativa entre as diferentes formulações (2 estudos analisados, 314 participantes), o gel mostrou-se mais eficaz que as demais [RR= 3,4 (IC95\%: 2,7-55); NNT=1,8 (IC95\%: 1,5-2,1)]. O cetoprofeno (7 estudos analisados; 683 participantes) também foi eficaz [RR=1,6 (IC95\%: 1,4-1,8); NNT=3,9 (IC95\%: 3,0-5,3)]. A formulação de cetoprofeno em gel foi mais eficaz que o emplastro [RR=2,2 (IC95\%: 1,7-2,8); NNT=2,5 (IC95\%: $2,0-3,4) ; z=3860 ; p=0,00014]$. Não havia dados suficientes para comparar de forma confiável os AINEs tópicos entre si, ou um mesmo AINE sob formulação tópica versus oral. As reações cutâneas locais (eritema e/ou prurido) foram geralmente leves e transitórias, e não diferiram do placebo. Poucos estudos relataram eventos adversos sistêmicos e não foi alcançada significância estatística [RR=0,96 (IC95\%: 0,73-1,3)]. Não houve relato de efeito adverso grave.

Dipirona. A dipirona (metamizol) é um AINE derivado da pirazolona, largamente empregado no Brasil no tratamento de dor e febre. Foi banida em diversos países, devido à ocorrência de reações alérgicas graves (como edema de glote e anafilaxia) e idiossincrásicas (agranulocitose potencialmente fatal) ${ }^{26,27}$. Uma das principais preocupações com a dipirona tem sido uma associação com agranulocitose. Existe uma vasta literatura sobre agranulocitose associada à dipi- rona. De maneira geral, sua frequência é baixa, com variação de dados entre diferentes autores. A predisposição genética é um fator descrito na agranulocitose induzida por dipirona ${ }^{23}$.

As eficácias dos antipiréticos dipirona, ibuprofeno e paracetamol foram comparadas em ensaio clínico randomizado ${ }^{31}$, duplo-cego, realizado em crianças febris ( $n=628$ ), com idade de seis meses a seis anos. Os resultados mostraram que os três medicamentos reduzem pelo menos $1,5^{\circ} \mathrm{C}$ em tempos semelhantes. Porém, a proporção de pacientes que alcançaram a normalização da temperatura (temperatura timpânica $\leq 37,5^{\circ} \mathrm{C}$ ) foi equivalente com dipirona (82\%) e ibuprofeno (78\%), e menor com paracetamol (68\%; $P=0,004)$. Dipirona teve efeito mais $d u-$ radouro, medido em quatro e seis horas. Não houve diferenças de efeitos adversos entre os três medicamentos.

Um ensaio clínico randomizado ${ }^{32}$ avaliou a eficácia de dipirona oral e intramuscular, e de ibuprofeno oral, em crianças com febre. O desfecho primário analisado foi a redução da temperatura corporal aos 30, 45, 60, 90 e 120 minutos depois da administração dos antipiréticos, combinada com banho de água em temperatura entre $32^{\circ} \mathrm{C}$ e $36^{\circ} \mathrm{C}$. Os resultados mostraram que a temperatura foi reduzida nos três grupos, em cerca de $0,5^{\circ} \mathrm{C}$, em 45 minutos, e $1,0^{\circ} \mathrm{C}$, em 120 minutos. Segundo os autores, a dipirona e o ibuprofeno têm perfil de segurança e eficácia semelhantes, atingindo a redução máxima da temperatura em 45 a 60 minutos.

Tendo igual eficácia e menor segurança do que outros antipiréticos, devido à imprevisibilidade de seus potenciais efeitos adversos, considera-se que a dipirona não deve ser primeira escolha em pacientes febris que possam ter acesso a alternativas igualmente eficazes e mais seguras. Haveria indicação apenas para o tratamento de febre elevada, não controlada 
por outras intervenções, ou em pacientes que não toleram outros antipiréticos ${ }^{23,27}$.

Revisão Cochrane ${ }^{33}$, envolvendo oito ensaios clínicos randomizados, duplo-cegos e controlados por placebo ( $n=809$ participantes), avaliou a eficácia e a segurança da dipirona em dose única no alívio da dor pós-operatória de intensidade moderada a grave. As buscas envolveram as bases de dados Medline, Cochrane Central Register of Controlled Trials, Oxford Pain Relief Database, LILACS e Embase, com artigos publicados até agosto de 2015. Para o desfecho pelo menos $50 \%$ de alivio da dor em quatro a seis horas após tratamento com dipirona 500 mg oral, o NNT foi de 2,4 (IC95\%: 1,9-3,1), RR=2,4 (IC95\%: 1,8-3,1), com evidência de qualidade moderada. O uso de medicação resgate foi menor com dipirona $500 \mathrm{mg}$ do que com placebo, com um NNT para prevenir um evento de 3,6 (IC95\%: 2,7-5,4), RR=0,21 (IC95\%: 0,11-0,4), com evidências de baixa qualidade. Esses dados sugerem que dose única de $500 \mathrm{mg}$ de dipirona proporciona alívio de ao menos $50 \%$ da intensidade da dor em período de quatro a seis horas para cerca de $73 \%$ de pacientes tratados, em comparação ao placebo. Os dados sobre o número de participantes que experimentaram qualquer efeito adverso foram relatados de forma inconsistente e nenhuma análise foi possivel. Mais comumente, a dipirona relacionou-se a sonolência, ao desconforto gástrico e a náusea.

Ibuprofeno. O ibuprofeno é um AINE que exibe atividades analgésica e antipirética ao inibir a síntese de prostaglandinas, e possui efeito inibitório sobre a agregação plaquetária. Em doses empregadas por automedicação, a incidência de eventos adversos gastrointestinais em adultos e crianças é similar à observada com paracetamol. Mesmo em doses mais elevadas, sob supervisão médica, ulceração e san- gramentos gastrointestinais são infrequentes (inferiores a 1\% dos casos), efeitos usualmente relacionados a tratamentos de longo prazo ${ }^{4,26,27}$.

É utilizado como substituto do paracetamol e do ácido acetilsalicílico no manejo de dores leves a moderadas, em variadas situações clínicas, e é um dos analgésicos sugeridos para o tratamento episódico de $\mathrm{CTT}^{27}$. Uma revisão sistemática ${ }^{12}$ envolvendo 12 estudos randomizados e controlados por placebo ( $n=1982$ participantes) avaliou a eficácia e a segurança do ibuprofeno oral no alívio sintomático da CTT episódica em adultos. As buscas envolveram as bases de dados Medline, The Cochrane Library e Embase, com artigos publicados até janeiro de 2015. Para o desfecho ausência de dor em duas horas, sem uso de medicação resgate, após tratamento com ibuprofeno 400 mg, o NNT foi de 14 (IC95\%: 8,4-47), RR=1,5 (IC95\%: 1,2-2,0), com evidência de qualidade moderada. O NNT de 5,9 (IC95\%: 4,2-9,5), RR=1,7 (IC95\%: 1,3-2,1) foi registrado para uma avaliação global de "muito bom" ou "excelente" (evidência de qualidade moderada). O uso de medicação de resgate foi menor com ibuprofeno $400 \mathrm{mg}$ do que com placebo, com um NNT para prevenir um evento de 8,9 (IC95\%: 5,6-21), RR=0,6 (IC95\%: 0,4-0,8), com evidências de baixa qualidade. Esses dados sugerem que o ibuprofeno, após duas horas do início do tratamento, na dose de 400 mg, alivia a CTT episódica em um pequeno número de pacientes. Os eventos adversos não foram diferentes entre ibuprofeno 400 mg e placebo, com evidência de alta qualidade. Não houve relato de evento adverso grave em qualquer dos estudos incluídos nesta revisão.

Um ensaio clínico ${ }^{34}(n=166)$ comparou a eficácia antipirética e a segurança de paracetamol $(15 \mathrm{mg} / \mathrm{kg})$ e ibuprofeno $(7 \mathrm{mg} / \mathrm{kg})$ no tratamento de crianças com idades de seis meses a 12 anos, com temperaturas corporais $\geq 38^{\circ} \mathrm{C}$. A temperatura foi registrada aos 60, 120,180 e 
240 minutos após a administração do medicamento, e 90\% das crianças tornaram-se afebris em algum momento durante o estudo com paracetamol e ibuprofeno. o índice máximo de redução da temperatura na amostra global foi alcançado durante os primeiros 60 minutos, depois da administração de ambos os fárma$\cos \left(-1,32 \pm 0,83^{\circ} \mathrm{C}\right.$ com ibuprofeno; $-1,09 \pm 0,77^{\circ} \mathrm{C}$ com paracetamol), mas sem diferença estatisticamente significante entre eles $(p=0,1)$. Em crianças entre cinco e 12 anos, o ibuprofeno proporcionou temperaturas significantemente mais baixas que o paracetamol $\left(38,00 \pm 0,65^{\circ} \mathrm{C}\right.$ vs $37,45 \pm 0,43^{\circ} \mathrm{C}, \mathrm{p}=0,02$, em 1 hora; $36,71 \pm 0,66^{\circ} \mathrm{C}$ vs $37,60 \pm 0,93^{\circ} \mathrm{C}, \mathrm{p}=0,01$, em duas horas; $36,80 \pm 0,79^{\circ} \mathrm{C}$ vs $37,67 \pm 1,12^{\circ} \mathrm{C}, \mathrm{p}=0,03$, em três horas). Os autores concluíram que o ibuprofeno e o paracetamol mostraram-se eficazes na redução da temperatura, exceto em crianças com mais de cinco anos, nas quais o ibuprofeno foi mais eficaz.

Naproxeno. O naproxeno é um AINE derivado do ácido propiônico com atividades analgésica, anti-inflamatória e antipirética. O mecanismo de ação envolve a inibição das ciclooxigenases COX-1 e COX-2, que leva à redução da sintese de prostaglandinas ${ }^{26}$. É utilizado no manejo de dor e inflamação em distúrbios musculoesqueléticos. Em relação a outros AINEs, o naproxeno apresenta eficácia similar e risco intermediário de reações adversas, especialmente gastrointestinais. Considerando dados clínicos e laboratoriais, naproxeno e paracetamol são vistos como aqueles com relação benefício-dano mais favorável no tratamento de dores agudas4.

O naproxeno é comumente empregado em tratamento de dor pós-operatória. Uma revisão sistemática ${ }^{35}$, envolvendo 15 estudos randomizados, duplo-cegos, controlados por placebo ( $n=1509$ participantes), avaliou a eficácia e a segurança do naproxeno oral, em dose única, no alívio da dor pós-operatória moderada a grave em adultos. A busca incluiu as bases de dados Medline, Cochrane Database, Oxford Pain Relief Database, The Cochrane Library e Embase, com artigos publicados até outubro de 2008. Para o desfecho 50\% de alívio da dor em quatro a seis horas, sem uso de medicação resgate, após uso de naproxeno 500 mg, o NNT foi de 2,7 (IC95\%: 2,3-3,2), RR=3,4 (IC95\%: 2,6-4,4). O tempo médio para uso de medicação resgate foi de 8,9 horas para o naproxeno e 2 horas para o placebo. O uso de medicação resgate foi significativamente menos comum com naproxeno do que com placebo. Os efeitos adversos associados foram geralmente de gravidade leve a moderada. Os resultados desta revisão sugerem que o naproxeno $500 \mathrm{mg}$ pode aliviar a dor pós-operatória em adultos, com quadro moderado a grave.

Nas bases de dados consultadas, não foram encontradas revisões sistemáticas que tenham avaliado o uso de naproxeno no tratamento da febre. As bulas dos medicamentos de referência do naproxeno no Brasil trazem, entre as indicações aprovadas pela Anvisa, o uso como analgésico e antipirético em adultos, como, por exemplo, nos sinais/sintomas de gripe e resfriado ${ }^{36}$. Ainda assim, em razão da falta de comprovação que apoie seu uso para tal finalidade, não se recomenda para o tratamento de febre supostamente de origem infecciosa ${ }^{19}$.

Paracetamol. O paracetamol é um analgésico e antipirético de ação central, com mínima propriedade anti-inflamatória. O mecanismo de ação do paracetamol na redução da dor é desconhecido, mas pode ser explicado pela inibição das ciclooxigenases, particularmente COX-2 e COX-3 no sistema nervoso central, e por uma elevação do limiar da dor. O paracetamol reduz a febre pela inibição da síntese de prostaglandinas no hipotálamo ${ }^{26}$. 
O paracetamol é um dos vários analgésicos sugeridos para o tratamento de CTT episódica frequente, moderada a intensa. Uma revisão sistemática ${ }^{37}$, envolvendo 23 estudos randomizados, duplo-cegos, controlados por placebo ( $n=8079$ participantes), avaliou a eficácia e a segurança do paracetamol oral no alívio sintomático da CTT episódica frequente em adultos. A busca incluiu as bases de dados Medline, Cochrane Database, Oxford Pain Relief Database e Embase, com artigos publicados até outubro de 2015. Foram incluídos todos os estudos que tinham pelo menos um grupo no qual o paracetamol foi administrado por via oral para o tratamento de CTT episódica, sem restrição de dose. O desfecho primário dessa revisão foi ausência de dor em duas horas, sem uso de medicação resgate. Os desfechos secundários incluíram ausência de dor sem uso de resgate em uma, quatro e 24 horas, após uso do paracetamol, dor leve ou ausência de dor em duas horas, uso de medicação resgate e eventos adversos. Para o desfecho ausência de dor em duas horas após o uso de paracetamol 1000 mg, o NNT foi de 22 (IC95\%: 15-40), RR=1,3 (IC95\%: 1,1-1,4), em oito estudos (5.890 participantes, com evidência de alta qualidade). O NNT foi de 10 (IC 95\%: 7,9-14), RR=1,2 (IC95\%: 1,15-1,3) para o desfecho ausência de dor ou dor leve em duas horas após o tratamento, e de 8,2 (IC95\%: 6,6-11) para ausência de dor em quatro horas. o uso de medicação resgate foi menos frequente com paracetamol $1000 \mathrm{mg}$ do que com placebo, obtendo-se um NNT=7,8 (IC 95\%: 6,0-11), RR=0,58 (IC95\%: 0,50-0,69), em seis estudos, para prevenir um evento. Dados limitados apontaram que a eficácia do paracetamol 500 mg e 650 mg não foi superior ao placebo, para alcançar o desfecho dor leve ou ausência de dor após duas horas e o paracetamol 1000 mg não foi diferente de cetoprofeno $25 \mathrm{mg}$ ou ibuprofeno 400 mg, com evidência de baixa qualidade.
A diferença de incidências de eventos adversos não foi estatisticamente significante entre paracetamol $1000 \mathrm{mg}$ e placebo [RR=1,1 (IC95\%: 0,94-1,3)] em 11 estudos (5605 participantes, evidências de alta qualidade). Dez estudos (5526 participantes) relataram eventos adversos gastrointestinais [RR=1,1 (IC 95\%: 0,86-1,5)] e quatro estudos (4036 participantes) relataram tontura como evento adverso [RR=1,5 (IC95\%: 0,83-2,6)]; no entanto, não houve diferença estatisticamente significativa entre paracetamol 1000 mg e placebo nesses resultados. Os estudos não relataram evento adverso grave. Para os pacientes com CTT episódica frequente, os resultados dessa revisão sugerem que o paracetamol 1000 mg pode aliviar a dor de cabeça, mas a chance da dor ser totalmente aliviada em duas horas é baixa.

Outra revisão sitemática Cochrane ${ }^{38}$, envolvendo 51 ensaios clínicos randomizados, duplo-cegos e controlados por placebo $(n=5762)$, avaliou a eficácia de dose oral única de paracetamol para o tratamento de dor aguda pós-operatória moderada a intensa em adultos. A busca incluiu as bases de dados Cochrane Library, Oxford Pain Relief Database, Medline e Embase, com artigos publicados até julho de 2008. Cerca de metade dos participantes tratados com paracetamol alcançaram pelo menos 50\% de alivio da dor em quatro a seis horas, em comparação com 20\% dos tratados com placebo [NNT=3,5 (IC95\%: 2,7-4,8), com dose de 500 mg; NNT=4,6 (IC95\%: 3,9-5,5), com doses de $600 \mathrm{mg}$ a 650 mg; NNT=3,6 (IC95\%: 3,4-4,0), com doses de 975 mg a 1000 mg]. A analgesia não se mostrou dependente da dose empregada. Cerca de metade dos participantes precisou de analgesia adicional ao longo de quatro a seis horas, em comparação com cerca de 70\% com o placebo. Para se evitar que um paciente precise de medicação resgate dentro de quatro a seis horas, 
foram obtidos os seguintes resultados: NNT=3,6 (IC95\%: 3,0-6,0), para dose de $500 \mathrm{mg}$ de paracetamol; NNT=7,8 (IC95\%: 5,2-15), para doses de 600 mg a 650 mg; e NNT=5,2 (IC95\%:4,3-6,7), para doses de 975 mg a 1000 mg. Os eventos adversos relatados foram principalmente leves e transitórios, com incidências semelhantes com 1000 mg de paracetamol e placebo. Não houve relato de evento adverso grave nos estudos incluídos nesta revisão.

O paracetamol é também muito utilizado no tratamento de febre em adultos e crianças. Uma revisão sistemática ${ }^{39}$, envolvendo 53 ensaios clínicos, randomizados e controlados ( $n=3155)$, avaliou a eficácia antipirética do paracetamol em doses de 10 a $15 \mathrm{mg} / \mathrm{kg}$, em crianças de até 12 anos. A busca foi realizada apenas na base de dados PubMed, envolvendo artigos publicados e não publicados até agosto de 2012. O paracetamol produziu uma resposta antipirética rápida, com diminuição da temperatura aparente em 30 minutos, e decréscimo máximo da temperatura corporal em $1,5^{\circ} \mathrm{C}$, no tempo médio de 2,8 horas, após a administração, se considerado o intervalo de quatro horas; e em $1,6^{\circ} \mathrm{C}$, no tempo médio de 3,4 horas, quando o intervalo de oito horas foi avaliado. Os eventos adversos mais comuns relatados nos estudos foram de natureza gastrointestinal e geralmente de intensidade baixa.

O paracetamol é agente de primeira escolha, por sua eficácia e maior segurança nas doses recomendadas, especialmente devido ao menor risco de sangramento e outros eventos gastrointestinais, em comparação a AINEs. Tem uso seguro em pacientes com história de doença péptica. Apesar de ser equivalente a ácido acetilsalicílico, prefere-se utilizá-lo em pacientes com possibilidade de efeitos adversos de salicilatos e em crianças com infecções virais. Pode ser prescrito a crianças, grávidas e idosos, na ausência de condição específica que o con- traindique. Em puérperas, é o analgésico não opioide mais indicado, por não acarretar efeitos indesejáveis ao lactente ${ }^{4,27}$.

No entanto, é o fármaco mais frequentemente envolvido em intoxicação farmacológica grave em diversos países, o que ressalta a necessidade de uso racional. As doses terapêuticas de paracetamol raramente causam efeitos adversos e a hepatotoxicidade é extremamente rara nessas condições ${ }^{4}$. Uma revisão sistemática ${ }^{40}$, envolvendo 791 estudos prospectivos e retrospectivos avaliou a ocorrência de insuficiência hepática, após o uso repetido de doses terapêuticas de paracetamol ( $4 \mathrm{~g} / \mathrm{dia}$ ou menos), por pelo menos 24 horas, em adultos. As buscas incluiram as bases de dados Medline e Embase. Dos 30.865 indivíduos incluídos em estudos prospectivos, 0,4\% apresentou nível de aminotransferase sérica que excedeu o limite normal, embora 13,8\% tenham usado a máxima dose terapêutica recomendada (3,9 a 4,0 g/dia). Não houve relato de insuficiência hepática ou morte. Em estudos retrospectivos ( $n=9.337), 1 \%$ dos pacientes apresentou nivel de alanina aminotransferase sérica que excedeu o limite normal, 0,01\% submeteu-se a transplante hepático e 0,06\% morreu. Alguns desses estudos apresentavam informações sugerindo que pacientes ingeriram doses altas de paracetamol, apesar de uma história de uso de doses terapêuticas.

Os pacientes devem ser orientados que não é seguro ultrapassar $4 \mathrm{~g}$ por dia e que diversas associações de venda livre contêm paracetamol. Há risco de dano hepático se quantidades excessivas do fármaco forem utilizadas. É contraindicado em pacientes com doença hepática ativa ou insuficiência hepática grave, podendo ser necessário redução da dose na insuficiência hepática leve a moderada, devido ao risco aumentado de lesão hepática. Alerta-se ainda que 
a sobredosagem pelo consumo escalonado (sobredoses em dias consecutivos) está associada a risco aumentado de hepatotoxicidade. O con- sumo de paracetamol concomitantemente com bebidas alcoólicas é também fator de risco para dano hepático e deve ser enfatizado ${ }^{28,41}$.

Quadro 2. Informações resumidas sobre os medicamentos antipiréticos e analgésicos isentos de prescrição.

\section{Fármaco: ÁCIDO ACETILSALICílICO}

\section{Considerações}

\section{Contraindicações:}

> Hipersensibilidade ao ácido acetilsalicílico ou a qualquer AINE.

> Asma, rinite ou pólipo nasal.

$>$ Crianças e adolescentes com menos de 16 anos (risco de Síndrome de Reye).

> Ulceração péptica prévia ou ativa.

$>$ Hemofilia e outras doenças hemorrágicas.

$>$ Insuficiência cardíaca grave.

$>$ Tratamento de gota.

Situações especiais:

$>$ Gravidez: evitar o uso no terceiro trimestre de gestação.

> Amamentação: uso criterioso. Risco em potencial da Síndrome de Reye.

> Insuficiência renal ou hepática: deve ser usado com cautela nos casos graves.

> Idosos: risco aumentado de sangramento, principalmente com doses acima de $325 \mathrm{mg} /$ dia por tempo prolongado.

Orientações aos pacientes:

> Orientar para ingerir o medicamento com alimentos ou grande quantidade de água para evitar desconforto gastrointestinal.

> Reforçar a importância de evitar o uso de bebida alcoólica.

> Estimular os pacientes a comunicarem os seguintes efeitos adversos: dor forte de estômago, vômito com sangue ou com aparência de grumos de café, sangue nas fezes ou urina, exantema ou bolhas na pele com prurido intenso, inchaço da face ou pálpebras, respiração dificil ou ruidosa, muita tontura ou sonolência, zumbido no ouvido.

\section{Fármaco: DICLOFENACO}

\section{Considerações}

Contraindicações:

$>$ Hipersensibilidade conhecida ao diclofenaco.

> Asma, angioedema, urticária ou rinite aguda.

Situações especiais:

> Gravidez: contraindicado durante o último trimestre de gestação.

Orientações aos pacientes:

> Não aplicar em lesões abertas, ou áreas irritadas (formulação para uso tópico). 


\section{Fármaco: DIPIRONA}

\section{Considerações}

Contraindicações:

$>$ Discrasias sanguíneas.

$>$ Supressão da medula óssea.

> Hipersensibilidade à dipirona, ao ácido acetilsalicílico ou aos AINEs.

$>$ Crianças com menos de três meses de idade ou menos de $5 \mathrm{~kg}$.

Situações especiais:

> Gravidez: uso não recomendado, principalmete durante o primeiro e o terceiro trimestres de gestação.

$>$ Amamentação: evitar o uso.

> Insuficiência renal: pode ser necessária a redução da dose em pacientes com nefropatia aguda.

Orientações aos pacientes:

> Orientar os pacientes a observarem desconforto gastrointestinal e relatarem história de ulceração, sangramento ou perfuração intestinal.

> Observar se o paciente encontra-se com infecção preexistente.

> Informar que em caso de dor e febre causadas por arboviroses, como dengue ou chicungunha, a dipirona ou o paracetamol devem ser os medicamentos de escolha.

\section{Fármaco: IBUPROFENO}

\section{Considerações}

Contraindicações:

$>$ Hemorragia gastrointestinal.

$>$ Úlcera gastrointestinal.

$>$ Insuficiência cardíaca grave.

$>$ Asma.

> Urticária ou outro tipo de reação de hipersensibilidade após administração de ibuprofeno, ácido acetilsalicílico ou outro AINE.

> No contexto da cirurgia de revascularização do miocárdio (by-pass).

Situações especiais:

> Gravidez: evitar o uso no terceiro trimestre de gestação.

> Amamentação: risco de dano ao lactente não pode ser descartado; evitar o uso.

> Insuficiência hepática: evitar o uso em doença hepática grave.

> Insuficiência renal: evitar o uso em doença renal grave.

> Idosos: evitar o uso prolongado, devido ao aumento do risco de sangramentos gastrointestinais, potencialmente fatais. Evitar o uso em idosos com insuficiência cardíaca (ICC) e com doença renal crônica, em estágio 4 ou 5.

Orientações aos pacientes:

> Estimular os pacientes a comunicar sinais/sintomas de problemas gastrointestinais graves (ex:: melena, hematêmese e/ou cólica intensa), reação cutânea (ex:: erupção bolhosa, eczema, urticária, coceira, exantema) ou de hepatotoxicidade (fadiga, letargia, icterícia, dor no quadrante superior direito do abdômen e sintomas gripais).

> Orientar a utilizar com comida para minimizar irritação gastrointestinal.

> Orientar a não consumir bebidas alcoólicas e nem fumar durante o tratamento. 


\section{Artigo Principal-}

\section{Fármaco: NAPROXENO}

\section{Considerações}

Contraindicações:

$>$ Hipersensibilidade ao naproxeno.

$>$ Hemorragia gastrointestinal ativa ou história prévia.

$>$ Histórico de perfuração gastrointestinal relacionada à terapia com AINE.

> Histórico de úlceras gastrointestinais recorrentes (dois ou mais episódios distintos).

$>$ Insuficiência cardíaca.

> Uso em cirurgia de enxerto do desvio de artéria coronária.

Situações especiais:

> Gravidez: evitar o uso a partir da trigésima semana, pois pode causar fechamento prematuro do canal arterial.

> Amamentação: risco ao lactente não pode ser descartado. Avaliar o risco-benefício.

> Insuficiência renal: uso não recomendado em doença renal moderada a grave.

> Idosos: evitar o uso prolongado, devido ao aumento do risco de sangramento gastrointestinal.

Orientações aos pacientes:

> Aconselhar os pacientes a reportarem sinais/sintomas de insuficiência cardíaca, de infarto, de eventos trombóticos, de derrame, de hepatotoxicidade (como hepatite e lesão hepática), de eventos gastrointestinais graves (hemorragias, úlceras ou perfuração), de reações cutâneas (exantema, dermatite esfoliativa, Síndrome de Stevens-Johnson ou necrólise epidérmica tóxica).

$>$ Informar que o medicamento pode causar tontura ou sonolência.

\section{Fármaco: PARACETAMOL}

\section{Considerações}

Contraindicações:

$>$ Hipersensibilidade ao paracetamol.

$>$ Doenças hepáticas grave e ativa.

$>$ Insuficiência hepática grave.

Situações especiais:

$>$ Gravidez: usar na gravidez somente se os beneficios potenciais para a mãe justificarem os riscos para o feto.

> Amamentação: risco mínimo ao lactente; compatível com a amamentação.

> Insuficiência hepática: redução da dose pode ser necessária na insuficiência hepática leve a moderada.

> Insuficiência renal: pode ser necessário aumentar o intervalo entre as doses ou reduzir a dose total.

Orientações aos pacientes:

$>$ Informar que não é seguro ultrapassar $4 \mathrm{~g}$ por dia.

> Alertar para a existência de diversas associações de venda livre contendo paracetamol. Risco de dano hepático se usar de modo concomitante.

> Orientar para a ingestão de comprimidos com um copo cheio de água.

$>$ Não ingerir bebida alcoólica, enquanto estiver em uso desse medicamento. Risco de dano hepático.

> Alertar para interromper o tratamento e procurar rapidamente atendimento médico, na ocorrência de exantema ou outras manifestações dermatológicas.

> Orientar que, em caso de dor e febre causadas por arboviroses, como dengue ou chicungunha, a dipirona ou o paracetamol devem ser os medicamentos de escolha

Fonte: Anvisa ${ }^{36}$, IBM Micromedex ${ }^{26}$, MedSus ${ }^{28}$. 


\section{Considerações finais}

A seleção da terapia farmacológica para manejo da dor e da febre deve levar em conta a eficácia, a segurança, a conveniência da forma farmacêutica e a preferência do paciente. As possiveis interações do medicamento "selecionado" com outros medicamentos, alimentos, tabaco e álcool também devem ser observadas. Devem ser analisadas com cautela as situações especiais, como gravidez, amamentação, insuficiência renal ou hepática, a fim de permitir a adoção da conduta mais adequada em cada caso e para cada paciente.

Os analgésicos não opioides são os medicamentos mais comumente utilizados no tratamento de dores leves a moderadas e da febre. 0 paracetamol aparece como agente de primeira escolha, para adultos e crianças, por sua eficácia e maior segurança nas doses recomendadas. O ácido acetilsalicílico é uma opção ao paracetamol, pois apresenta igual eficácia analgésica e antipirética. No entanto, apresenta menor segu- rança devido à associação causal com a síndrome de Reye em crianças com infecções virais e à ocorrência de sangramento em pacientes com dengue, zica e chicungunha.

O ibuprofeno, em doses empregadas por automedicação, apresenta incidência de eventos adversos gastrointestinais em adultos e crianças similar à observada com paracetamol, sendo utilizado como substituto do paracetamol e do ácido acetilsalicílico no manejo de dores leves a moderadas. O naproxeno apresenta eficácia similar e risco intermediário de reações adversas em relação a outros AINEs, mas seu uso como antipirético ainda é questionável em razão de falta de comprovação científica que apoie essa indicação. A dipirona foi banida em diversos países, devido à ocorrência de anafilaxia, edema e agranulocitose potencialmente fatal. Deve-se ter cautela ao utilizar em virtude da imprevisibilidade de seus potenciais efeitos adversos.

\section{Referências}

1. Vidyavati S, Sneha A, Kamarudin J, Katti S. Self Medication Reasons, Risks and Benefits. International J of Healthcare and Biomedical Research. 2016;04(04):21-4.

2. Matos JF et al. Prevalence, profile and factors associated with self-medication in adolescents and employees of a professionalizing public school. Cad. Saúde Colet. 2018;26(1):76-83

3. Agência Nacional de Vigilância Sanitária. Instrução Normativa n 86, de 12 de março de 2021. Diário Oficial da União.17 mar 2021;Seção 1.

4. Fuchs FN, Wannmacher L. Farmacologia Clínica. Fundamentos da terapêutica Racional. 5. ed. Rio de Janeiro: Guanabara Koogan; 2017.

5. Yeng LT, Teixeira MJ. Tratamento multidisciplinar dos doentes com dor crônica. [acesso em 9 fev 2021]. Disponivel em: http://celiagagliardi.com.br/pdf/entrevista.pdf.

6. World Health Organization (WHO). WHO's Cancer pain ladder for adults. [acesso em 10 jan 2021]. Disponivel em: http://www.who.int/cancer/palliative/painladder/en/.

7. Breivik H, Borchgrevink PC, Allen SM et al. Assesment of pain. British Journal of Anaesthesia. 2008;101(1):17-24.

8. Stovner LJ, Hagen K, Jensen R, Katsarava Z, Lipton R, Scher Al, et al. The global burden of headache: a documentation of headache prevalence and disability worldwide. Cephalalgia 2007;27(3):193-210.

9. Headache Classification Committee of the International Headache Society (IHS). The international classification of headache disorders. 3rd edition. 2018;38(1):1-211.

10. Hoefler R, Moura AN. Cefaleia por uso excessivo de medicamento. Boletim Farmacoterapêutica. 2012;3:9-15.

11. Sociedade Brasileira para o Estudo da Dor (SBED). Campanha do ano mundial contra a dor de cabeça. Outubro 2011-outubro 2012. Cefaleia por uso excessivo de medicamento. [acesso em 9 fev 2021]. Disponivel em: https://sbed.org.br

12. Derry S, Wiffen PJ, Moore RA, Bendtsen L. Ibuprofen for acute treatment of episodic tension-type headache in adults. Cochrane Database Syst Rev. 2015;(7):CD011474 
13. Herzog L, Phillips SG. Addressing concerns about fever. Clin. Pediatr. 2011;50:383-390.

14. Gradiz R, Pinto AM. Fisiopatologia-Fundamentos e Aplicações. Lidel, Edições Técnicas. 2013;233-42.

15. Brunton LL, Lazo JS, Parker KL. Goodman e Gilman: As bases farmacológicas da terapêutica. 11a edição. Rio de Janeiro: McGraw-Hill Interamericana do Brasil; 2006.

16. Garcia-Zapata MTA, Júnior ESS. Aspectos fisiopatológicos da febre nas doenças infecto-parasitárias. 2006;4(1/2).

17. Rang HP, Dale MM, Ritter JM. Farmacologia. 4a edição. Ed. Guanabara Koogan. Rio de Janeiro: Rio de Janeiro; 2001.

18. Kasper DL, Fauci A, Hauser S, Longo D, Jameson L, Loscalzo J. Fever. In: Fauci AS, editor. Harrison's manual of medicine. 19. ed. New York: The McGraw-Hill Companies; 2016. chapter 28.

19. Conselho Federal de Farmácia. Guia de prática clinica: sinais e sintomas não específicos: febre. Brasília: Conselho Federal de Farmácia; 2018.

20. Bruni O, Russo PM, Ferri R, Novelli L, Galli F, Guidetti V. Relationships between headache and sleep in a nonclinical population of children and adolescents. Sleep Med. 2008;9(5):542-8.

21. Tate A, Tuerner GN, Knab SE, Jorgensen C, Strittmatter A, Michener LA. Risk factors associated with shoul der pain and disability across the lifespan of competitive swimmers. Journal of Athletic Training. 2012;47(2):149-58.

22. Speciali JG, Kowacs F, Jurno ME, Bruscky IS, Carvalho JJF, Francisca Goreth Malheiro, Fantini FGMM, et al. Protocolo nacional para diagnóstico e manejo das cefaleias nas unidades de urgência do Brasil-2018. São Paulo: Academia Brasileira de Neurologia-Departamento Científico de Cefaleia;2018.

23. Wannmacher L, Ferreira MBC. Febre: mitos que determinam condutas. Uso racional de medicamentos: temas selecionados. Brasília: Uso Racional de Medicamentos: temas Selecionados. 2004;1(9):1-9. [acesso em 2 fev 2021]. Disponivel em: <http://www.ibilce.unesp.br

24. Meremikwu M, Oyo-Ita A. Physical methods for treating fever in children. Cochrane Database Syst Rev. 2003; 2:CD004264.

25. Ong CK, Lirk P, Tan CH, Seymour RA. An evidence-based update on nonsteroidal antiinflammatory drugs. Clin Med Res. 2007;5(1):19-34

26. IBM Micromedex ${ }^{\circledR}$ [internet]. Greenwood Village: IBM Watson Health [acesso em 2 mar 2021]. Disponivel em: https://www.micromedexsolutions.com/

27. Brasil. Ministério da Saúde. Secretaria de Ciência, Tecnologia e Insumos Estratégicos. Departamento de Assistência Farmacêutica e Insumos Estratégicos. Formulário terapêutico nacional 2010: Rename 2010. 2. ed. Brasília: Ministério da Saúde, 2010.
28. MedSUS [internet]. Medicamentos Indicados pelo SUS. Ministério da Saúde. Android (2021) [Aplicativo móvel]. Disponivel em: Play Store.

29. Derry S, Moore RA. Single dose oral aspirin for acute postoperative pain in adults. Cochrane Database Syst Rev. 2012;(4):CD002067.

30. Derry S, Moore RA, Gaskell H, Mclntyre M, Wiffen PJ. Topical NSAIDs for acute musculoskeletal pain in adults. Cochrane Database Syst Rev. 2015;(6):CD007402.

31. Wong A, Sibbald A, Ferrero F, Plager $M$, Santolaya ME, Escobar AM, et al. Fever Pediatric Study Group. Antipyretic effects of dipyrone versus ibuprofen versus acetaminophen in children: results of a multinational, randomized, modified double-blind study. Clin Pediatr (Phila). 2001;40(6):313-24.

32. Prado J, Daza R, Chumbes O, Loayza I, Huicho L. Antipyretic efficacy and tolerability of oral ibuprofen, oral dipyrone and intramuscular dipyrone in children: a randomized controlled trial. Sao Paulo Med J. 2006;124(3):135-40.

33. Hearn L, Derry S, Moore RA. Single dose dipyrone (metamizole) for acute postoperative pain in adults. Cochrane Database Syst Rev. 2016;(4):CD011421.

34. Aguado IC, López IJ, Pinilla MLC, García IC, Lázaro AMP, Bengoechea PB et al. Antipyretic effectiveness of ibuprofen and paracetamol. Ann. Pediatr. (Barc.). 2005;62(2):117-22.

35. Derry CJ, Derry S, Moore RA, McQuay HJ. Single dose oral naproxen and naproxen sodium for acute postoperative pain in adults. Cochrane Database Syst Rev. 2009;(1): CD004234

36. Agência Nacional de Vigilância Sanitária (Anvisa) [internet]. [acesso em 14 mar 2021]. Disponível em https://www.gov.br/anvisa/pt-br

37. Stephens G, Derry S, Moore RA. Paracetamol (acetaminophen) for acute treatment of episodic tension-type headache in adults. Cochrane Database Syst Rev. 2016; (6): CD011889.

38. Toms L, McQuay HJ, Derry S, Moore RA. Single dose oral paracetamol (acetaminophen) for postoperative pain in adults. Cochrane Database Syst Rev. 2008;(4):CD004602.

39. Temple AR, Temple BR, Kuffner EK. Dosing and antipyretic efficacy of oral acetaminophen in children. Clinical therapeutics. 2013;35(9):1361-75.

40. Dart RC, Bailey E. Does therapeutic use of acetaminophen cause acute liver failure? Pharmacotherapy. 2007;27(9): 121930.

41. Craig DG, Bates CM, Davidson JS, Martin KG, Hayes PC, Simpson KJ. Staggered overdose pattern and delay to hospital presentation are associated with adverse outcomes following paracetamol-induced hepatotoxicity. Br J Clin Pharmacol. 2012;73(2):285-94. 